\title{
SIGNATURES OF HADRONIC COSMIC RAYS IN STARBURSTS? HIGH-ENERGY PHOTONS AND NEUTRINOS FROM NGC 253
}

\author{
Gustavo E. Romero ${ }^{1}$ and Diego F. Torres ${ }^{2}$ \\ Received 2002 December 27; accepted 2003 February 7; published 2003 February 21
}

\begin{abstract}
We show that it appears possible for starburst galaxies, like the nearby NGC 253 , recently identified as a TeV source by the CANGAROO collaboration, to emit a significant amount of high-energy $\gamma$-rays and neutrinos through hadronic processes in their cores. We suggest that proton illumination of the inner winds of massive stars can be a viable mechanism for producing $\mathrm{TeV} \gamma$-rays and neutrinos without a strong $\mathrm{MeV}-\mathrm{GeV}$ counterpart. The rich stellar content of the starbursts, with millions of early-type stars concentrated in the central regions, where collective effects of the stellar winds and supernovae can produce a significant enhancement of the cosmicray density, provides an adequate scenario for $\mathrm{TeV} \gamma$-ray generation. Close starbursts are also found to be potential sources for kilometer-scale neutrino telescopes, like IceCube, within reasonable integration times.
\end{abstract}

Subject headings: galaxies: individual (NGC 253) — galaxies: starburst — gamma rays: observations gamma rays: theory

\section{INTRODUCTION}

Very recently, the CANGAROO collaboration reported the detection of the nearby starburst galaxy NGC 253 at TeV $\gamma$-ray energies (Itoh et al. 2002). The purpose of this Letter is to show that it is possible for starburst galaxies, like NGC 253, to produce $\mathrm{TeV}$ photons and neutrinos through hadronic processes.

\section{A PLAUSIBLE SCENARIO}

Collective effects of strong stellar winds and supernova explosions in star-forming regions are expected to result in particle acceleration up to multi-TeV energies, or even beyond (e.g., Montmerle 1979; Cassé \& Paul 1980; Bykov \& Fleishman 1992a, 1992b; Anchordoqui, Romero, \& Combi 1999; Bykov 2001). Recently, the region cospatial with the Cyg OB2 association has been detected as a $\mathrm{TeV} \gamma$-ray source by the High Energy Gamma Ray Astronomy telescope array (Aharonian et al. 2002). $\mathrm{TeV}$ cosmic rays (CRs) accelerated in the association might be responsible for the high-energy $\gamma$-ray emission through the hadronic illumination of some suitable target (Butt et al. 2003). A nearby EGRET source (3EG J2033+4118) has also a likely stellar origin (Chen, White, \& Bertsch 1996; Romero, Benaglia, \& Torres 1999; Benaglia et al. 2001).

In a starburst, where the ambient density of CRs is enhanced by a local high rate of supernova explosions and the collective effects of strong stellar winds (e.g., Bykov 2001), $\gamma$-rays can be produced by interactions of relativistic protons with the rich interstellar medium. This interaction produces neutral pions that quickly decay into $\gamma$-rays. The latter can also be produced by leptonic processes, like inverse Compton interactions with the strong far-infrared (FIR) field, and relativistic bremsstrahlung in the ambient gas. All these processes have been modeled with a set of reasonable parameters for NGC 253 by Paglione et al. (1996). The expected total $\gamma$-ray flux, however, is below the EGRET sensitivity limit, in accordance with the nondetection of the galaxy in the MeV-GeV range (Bhattacharya et al. 1994; Blom, Paglione, \& Carramiñana 1999).

A source of $\gamma$-rays not previously considered in the men-

\footnotetext{
${ }^{1}$ Instituto Argentino de Radioastronomía, Casilla de Correo 5, 1894 Villa Elisa, Argentina; romero@irma.iar.unlp.edu.ar.

${ }^{2}$ Lawrence Livermore National Laboratory, 7000 East Avenue, L-413, Livermore, CA 94550; dtorres@igpp.ucllnl.org.
}

tioned analysis is the production at the base of the strong stellar winds of early-type stars. When the relativistic particles are accelerated in the stellar wind itself, for instance, through multiple shocks produced by line-driven instabilities (e.g., White $\&$ Chen 1992), the $\gamma$-ray flux from pion decays is limited to $\mathrm{MeV}$ energies. However, if the star is immersed in an external bath of relativistic particles accelerated at larger scales, e.g., in the core of the starburst, $\mathrm{TeV}$ protons can penetrate the base of the wind to produce $\mathrm{TeV} \gamma$-rays. The injection of $\mathrm{MeV}-\mathrm{GeV}$ particles into the wind of the stars will be strongly attenuated by the modulation effects of the wind itself.

The dense medium in which these $\gamma$-rays are produced is, nonetheless, transparent to $\gamma$-ray propagation. The optical depth to pair production is $\tau \sim n \sigma R$, where $n$ is the photon number density, $\sigma$ is the photon-photon cross section, and $R$ is the distance that the photon must travel to escape. In order to compute $n$, we need the photon energy density at the base of the stellar wind, $U$. We shall assume that target photons in the wind have typical energies of $\sim 1 \mathrm{eV}$. Then, $U=3 L /\left(4 \pi R^{2} c\right) \sim 2 \times 10^{10} \mathrm{eV} \mathrm{cm}^{-3}$, where we have taken, for order-of-magnitude estimates, the following typical values: $R \sim 5 \times 10^{14} \mathrm{~cm}$ and $L \sim 10^{39} \mathrm{ergs} \mathrm{s}^{-1}$. Then, $n=U /(1 \mathrm{eV})$, and the optical depth is very small, $\tau \sim$ $2 \times 10^{-5}$. The computation of $\tau$ makes use of the $\gamma-\gamma(1 \mathrm{TeV}-$ $1 \mathrm{eV}$ ) cross section, $\sigma \sim 2 \times 10^{-30} \mathrm{~cm}^{2}$ (Lang 1999, p. 434). Once $\mathrm{TeV}$ photons are produced and escape from the wind region, they must yet leave the galaxy to reach the Earth. The starburst has an FIR field with $U \sim 200 \mathrm{eV} \mathrm{cm}^{-3}$ (e.g., Paglione et al. 1996), and photons have to travel distances of about 70 $100 \mathrm{pc}$ to leave the active region of the galaxy. The optical depth is, in this case, $\tau=\left(200\right.$ photons $\left.\mathrm{cm}^{-3}\right)\left(2 \times 10^{-30} \mathrm{~cm}^{2}\right) \times$ $(100 \mathrm{pc}) \approx 1.2 \times 10^{-7}$, which is also small enough to secure that photons can escape from the galaxy.

\section{NGC 253}

NGC 253 has been described as an archetypal starburst galaxy by Rieke et al. (1980), and it has been extensively studied from radio to $\gamma$-rays (e.g., Beck et al. 1994; Paglione et al. 1996; Ptak et al. 1997). The supernova rate is estimated to be as high as $0.2-0.3 \mathrm{yr}^{-1}$, comparable to the massive star formation rate, $\sim 0.1 M_{\odot} \mathrm{yr}^{-1}$ (Ulvestad \& Antonucci 1997; Forbes et al. 1993). The central region of this starburst is packed with massive stars. Watson et al. (1996) have discovered four young globular clusters 
near the center of NGC 253; they alone can account for a mass well in excess of $1.5 \times 10^{6} M_{\odot}$ (see also Keto et al. 1999). Assuming that the star formation rate has been continuous in the central region for the last $10^{9} \mathrm{yr}$, and a Salpeter initial mass function for $0.08-100 M_{\odot}$, Watson et al. (1996) find that the bolometric luminosity of NGC 253 is consistent with $1.5 \times$ $10^{8} M_{\odot}$ of young stars. Based on this evidence, it appears likely that there are at least tens of millions of young stars in the central region of the starburst. Many stars might be obscured by the large amount of molecular material, as in the case of the massive Galactic cluster Westerlund 1 (Clark \& Negueruela 2002).

Physical, morphological, and kinematic evidence for the existence of a galactic superwind has been found for NGC 253 (e.g., McCarthy, Heckman, \& van Breugel 1987; Heckman, Armus, \& Miley 1990). This superwind creates a cavity of hot $\left(\sim 10^{8} \mathrm{~K}\right)$ gas, with cooling times longer than the typical expansion timescales. As the cavity expands, a strong shock front is formed on the contact surface with the cool interstellar medium. The shock velocity can reach thousands of kilometers per second, and iron nuclei can be efficiently accelerated up to high energies $\sim 10^{20} \mathrm{eV}$ by diffusive mechanisms operating at large scales. Indeed, NGC 253 has been proposed as the origin for some of the observed ultra-high-energy CRs (Anchordoqui et al. 1999; Anchordoqui, Goldberg, \& Torres 2002).

The CANGAROO collaboration did not give a precise photon spectrum for NGC 253. The reported data were only the integrated flux above $0.5 \mathrm{TeV}$ (securing an $11 \sigma$ confidence detection) and the possible range of power-law indices $\Gamma=(1.85,3.75)$ (Itoh et al. 2002). We can summarize these results expressing the differential photon spectrum as $F_{\gamma}\left(E_{\gamma}\right)=B\left(E_{\gamma} / 1 \mathrm{TeV}\right)^{-\Gamma}$ $\mathrm{cm}^{-2} \mathrm{~s}^{-1} \mathrm{TeV}^{-1}$, where $B=(3.8 \pm 1.2) \times 10^{-12}$ for $\Gamma=1.8$, or $B=(3.2 \pm 1.0) \times 10^{-12}$ for $\Gamma=3.75$. It is worth noticing that the direct extrapolation of the spectrum at $\mathrm{TeV}$ energies down to the $\mathrm{GeV}$ band would yield an integrated value compatible with the EGRET upper limits for NGC 253 only for power-law indices $\Gamma \lesssim 2$. Spectra softer than those with $\Gamma=$ 2 at $\mathrm{TeV}$ energies should present a break at a few hundred $\mathrm{GeV}$.

\section{HADRONIC ORIGIN OF THE TeV EMISSION FROM NGC 253?}

The differential $\gamma$-ray photon number distribution from neutral pion decays at the source is given by (e.g., Gaisser 1990) $N_{\gamma}\left(E_{\gamma}\right)=N_{p}\left(E_{\gamma}\right) \xi_{A} 4 \pi \sigma_{p p}\left(E_{\gamma}\right) N 2 Z_{p \rightarrow \pi^{0}}^{(\alpha)} / \alpha$. Here $E_{\gamma}$ is the photon energy, $N=\Sigma / m_{p}$ is the column density, $m_{p}$ is the proton mass, and $\alpha$ is the proton spectral index, such that $N_{p}=$ $K_{p} E^{-\alpha}$. In addition, $\xi_{A} \sim 1.5$ is a correction factor that takes into account possible effects of heavier nuclei, $\sigma_{p p}\left(E_{\gamma}\right) \sim$ 35 mbarn is the hadronic interaction cross section, and $Z_{p \rightarrow \pi^{0}}^{(\alpha)} \sim 0.17$ stands for the fraction of kinetic energy of the parent proton that is transferred to the neutral pion during the collision. For a photon/proton spectral index $\alpha \sim 2$, we get $N_{\gamma}\left(E_{\gamma}\right)=1.12 \times 10^{-25} K_{p} E_{\gamma}^{-2} \mathrm{~cm}^{-3} \mathrm{TeV}^{-1}$ (notice that $1 \mathrm{erg} \sim 1 \mathrm{TeV})$. Assuming typical grammages for the stellar winds of OB stars in the range $\Sigma \sim 50-150 \mathrm{~g} \mathrm{~cm}^{-2}$ (e.g., White 1985), we get $N_{\gamma}\left(E_{\gamma}\right)=(3.4-10.1) K_{p} E_{\gamma}^{-2} \mathrm{~cm}^{-3} \mathrm{TeV}^{-1}$. The luminosity in the CANGAROO observing range is

$$
\begin{aligned}
L & =4 \pi R^{2} c \int_{\sim 0.5 \mathrm{TeV}}^{\sim 20 \mathrm{TeV}} N_{\gamma}\left(E_{\gamma}\right) E_{\gamma} d E_{\gamma} \\
& =(4.7-13.9) \times 10^{12} R^{2} K_{p} \text { ergs s}{ }^{-1},
\end{aligned}
$$

where $R$ is the size of the base of the wind and $c$ is the speed of light (all numerical coefficients are for cgs units). The CR energy density is, on the other hand,

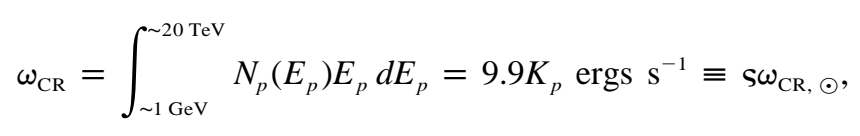

where $s$ is the amplification factor of the CR energy density with respect to the local value, $\omega_{\mathrm{CR}, \odot} \sim 10^{-12} \mathrm{ergs}^{-3}$. This implies that the $\mathrm{TeV}$ luminosity can be written as $L=$ $(0.47-1.4) R^{2} s \mathrm{ergs} \mathrm{s}^{-1}$. The latter corresponds to the luminosity of a single star illuminated by TeV CR protons. In the central region of the starburst, millions of stars could be illuminated at the same time. The total luminosity would then be $L_{\text {total }}=\sum_{i=1}^{N_{*}}(0.47-1.4) R_{i}^{2} \varsigma_{i}$ ergs s${ }^{-1}$, where $N_{*}$ is the number of contributing stars and we have allowed for the possibility that not all of them will have the same $R$, nor will they be located within regions having the same $\mathrm{CR}$ enhancement factor.

If we now assume the following ranges for the different parameters involved-1 $1 \times 10^{12}<R(\mathrm{~cm})<1 \times 10^{15}$ (Lamers \& Cassinalli 1999), $300<s<3000$ (Suchkov, Allen, \& Heckman 1993), and $10^{6}<N_{*}<5 \times 10^{7}$ (see above)-there is ample room for the total luminosity to reach values above $10^{39}$ ergs $\mathrm{s}^{-1}$, i.e., the observed CANGAROO luminosity above $0.5 \mathrm{TeV}$, given the distance to NGC 253 (2.5 Mpc; de Vaucouleurs 1978). To show this, we have carried out Monte Carlo numerical simulations where the number of stars illuminated by the accelerated protons, the grammage, the size of the base of the wind for each star, and the ambient enhancement of CRs are independently allowed to take values within their assumed ranges. For each of these cases, the total luminosity was computed. We show the results in Figure $1 a$, for a sample of 1000 trials. Only for less than $40 \%$ of the trials, the total luminosity is below $10^{39} \mathrm{ergs} \mathrm{s}^{-1}$. This scenario then appears to be a plausible explanation for the TeV observations of NGC 253.

Note that multi-TeV electrons in the starburst central region will experience inverse Compton losses with the FIR fields (typical energy density $\sim 200 \mathrm{eV} \mathrm{cm}^{-3}$ ) in the Klein-Nishina regime, which result in a very steep $\gamma$-ray spectrum (that can be approximated by power-law indices $\gtrsim 3.2$, although notice that the actual spectrum is not a pure power law; see, e.g., Georganopoulos, Kirk, \& Mastichiadis 2001). The total leptonic contribution from the central starburst region at $\mathrm{TeV}$ energies is then negligible in comparison to the hadronic one.

$\mathrm{MeV}-\mathrm{GeV}$ particles cannot easily penetrate the stellar winds, and hence these winds will glow mainly at $\mathrm{TeV}$ energies. The modulation of CRs by stellar winds is a complex subject that has been studied in detail only in the context of the relatively weak solar wind. In such case, three-dimensional models including diffusion and the effects of the terminal shocks have been developed (e.g., Kóta \& Jokipii 1983; Jokipii, Kóta, \& Merényi 1993). These models can explain fairly well the basic observed features of the nucleonic CR component in the solar system, where strong gradients in the CR density are known to exist from interplanetary spacecraft measurements (e.g., Quenby, Lockwood, \& Webber 1990). ${ }^{3}$ In OB stars, with extremely supersonic winds and mass-loss rates orders of magnitude higher than the solar values, these effects should be far more pro-

\footnotetext{
${ }^{3}$ Although it is highly dependent on the solar activity and the inclination angle of the incident particles, the proton flux decreases to less than $\sim 30 \%$ of its initial value at $80 \mathrm{AU}$ for $2.5 \mathrm{GeV}$ particles and to less than $10 \%$ for $250 \mathrm{MeV}$ particles.
} 

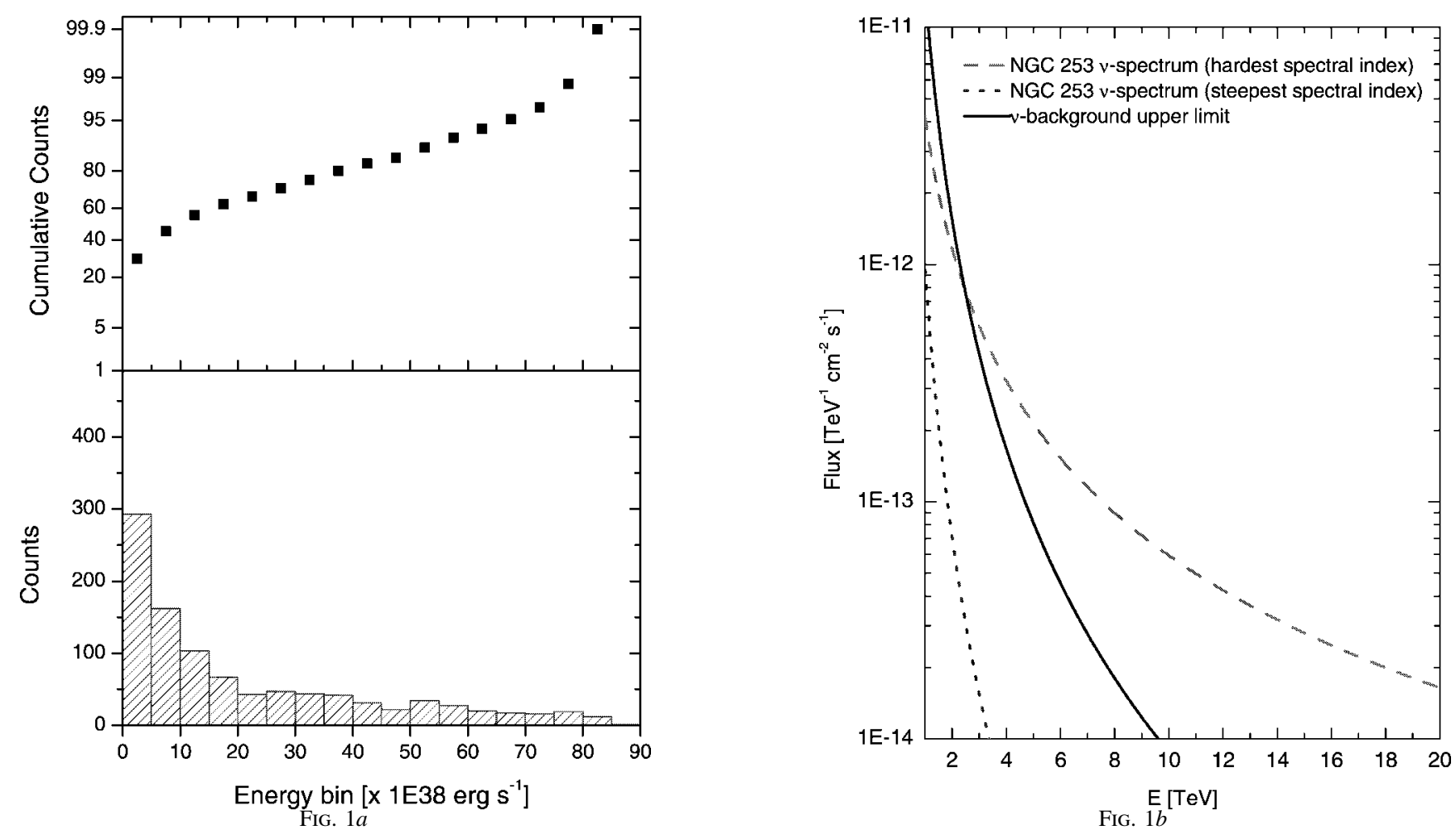

FIG. 1.-(a) Results of 1000 Monte Carlo trials to compute the total TeV luminosity of a starburst lying at the distance of NGC 253 . (b) Hadronic model expectation of neutrinos from NGC 253 compared with the upper limit for the neutrino background in a $1^{\circ} \times 1^{\circ}$ generation neutrino telescope.

nounced, leading to an almost complete rejection of externally injected $\mathrm{MeV}-\mathrm{GeV}$ particles at the base of the winds and thus to an absence of a strong (above EGRET sensibility) $\gamma$-ray counterpart at these energies. The modulation effect depends on the parameter $\epsilon \sim u R_{w} / D$, where $u$ is the stellar wind velocity, $R_{w}$ is the radius of the wind shell, and $D$ is the diffusion coefficient. The parameter $\epsilon$ measures the ratio between the diffusive timescale of the particle to their convective timescale. The former should be shorter than the latter for a $\mathrm{TeV}$ particle to enter into the wind cavity. For a typical $\mathrm{O}$ star, $R_{w}>10 \mathrm{pc}$ and $u \sim$ $2500 \mathrm{~km} \mathrm{~s}^{-1}$. Hence, the diffusion coefficient of the $\mathrm{TeV}$ particles should be at least $10^{28} \mathrm{~cm}^{2} \mathrm{~s}^{-1}$, similar to what is estimated for our Galaxy (e.g., Berezinskii et al. 1990).

\section{NEUTRINO SIGNAL}

If the $\gamma$-ray emission is explained by the decay of neutral pions, their charged counterparts must produce a neutrino flux. Following Alvarez-Muñiz \& Halzen (2002), the $\nu_{\mu}+\bar{\nu}_{\mu}$ neutrino flux $F_{v}\left(E_{\nu}\right)$ produced by the decay of charged pions in the source can be derived from the observed $\gamma$-ray flux $F_{\gamma}\left(E_{\gamma}\right)$ by imposing energy conservation (see also Stecker 1979):

$$
\int_{E_{\gamma}^{\min }}^{E_{\gamma}^{\max }} E_{\gamma} F_{\gamma}\left(E_{\gamma}\right) d E_{\gamma}=K \int_{E_{\nu}^{\min }}^{E_{\nu}^{\max }} E_{\nu} F_{\nu}\left(E_{\nu}\right) d E_{\nu} .
$$

Here $E_{\gamma(\nu)}^{\min }\left(E_{\gamma(\nu)}^{\max }\right)$ is the minimum (maximum) energy of the photons (neutrinos) and the prefactor $K=1 .^{4}$ The maximum

\footnotetext{
${ }^{4}$ Note that there are two muon-neutrinos (out of the pion-to-muon-to-electron decay chain) for every photon produced. Each of the latter carries, on average, an energy $E_{\pi} / 4$, whereas each photon carries on average half the energy of the pion. Therefore, there is as much energy in photons as there is in neutrinos, and $K=1$.
}

neutrino energy is fixed by the maximum energy of the accelerated protons $\left(E_{p}^{\max }\right)$, which can in turn be obtained from the maximum observed $\gamma$-ray energy $\left(E_{\gamma}^{\max }\right)$ as $E_{p}^{\max } \sim 6 E_{\gamma}^{\max }$, $E_{\nu}^{\max } \sim 1 / 12 E_{p}^{\max }$ (Alvarez-Muñiz \& Halzen 2002). The minimum neutrino energy is fixed by the threshold for pion production, $E_{p}^{\min }=\delta\left[\left(2 m_{p}+m_{\pi}\right)^{2}-2 m_{p}^{2}\right] / 2 m_{p}$, where $\delta$ is the Lorentz factor of the accelerator relative to the observer. Assuming a Lorentz factor of order 1 , the $\nu$-spectrum results in $F_{v}\left(E_{\nu}\right) \sim 4.2 \times 10^{-12}(E / \mathrm{TeV})^{-1.85} \mathrm{TeV}^{-1} \mathrm{~cm}^{-2} \mathrm{~s}^{-1}$ for the harder photon index and $F_{\nu}\left(E_{\nu}\right) \sim 9.5 \times 10^{-13}(E / \mathrm{TeV})^{-3.75}$ $\mathrm{TeV}^{-1} \mathrm{~cm}^{-2} \mathrm{~s}^{-1}$ for the softer photon index.

The event rate of atmospheric $\nu$-background is (see Anchordoqui et al. 2003)

$$
\left|\frac{d N}{d t}\right|_{B}=A_{\text {eff }} \int d E_{\nu} \frac{d \Phi_{B}}{d E_{\nu}} P_{\nu \rightarrow \mu}\left(E_{\nu}\right) \Delta \Omega_{1^{\circ} \times 1^{\circ}}
$$

where $A_{\text {eff }}$ is the effective area of the detector, $\Delta \Omega_{1^{\circ} \times 1^{\circ}} \approx$ $3 \times 10^{-4} \mathrm{sr}$ is an assumed bin size for the observation (IceCube-generation detector; Karle et al. 2002), and $d \Phi_{B} / d E_{\nu} \lesssim 0.2\left(E_{\nu} / \mathrm{GeV}\right)^{-3.21} \mathrm{GeV}^{-1} \mathrm{~cm}^{-2} \mathrm{~s}^{-1} \mathrm{sr}^{-1}$ is the $\nu_{\mu}+\bar{\nu}_{\mu}$ atmospheric $\nu$-flux (Volkova 1980; Lipari 1993). Here $P_{\nu \rightarrow \mu}\left(E_{\nu}\right) \approx 1.3 \times 10^{-6}\left(E_{\nu} / \mathrm{TeV}\right)^{0.8}$ denotes the probability that a $\nu$ of energy $E_{\nu}>1 \mathrm{TeV}$, on a trajectory through the detector, produces a muon (Gaisser, Halzen, \& Stanev 1995). On the other hand, the $\nu$-signal is

$$
\left|\frac{d N}{d t}\right|_{S}=A_{\text {eff }} \int d E_{\nu} F_{\nu}\left(E_{\nu}\right) P_{\nu \rightarrow \mu}\left(E_{\nu}\right)
$$

The signal-to-noise ratio $(\mathrm{S} / \mathrm{N})$ in the $1-20 \mathrm{TeV}$ band is then $\left(|d N / d t|_{S}\right) /\left(|d N / d t|_{B}\right)^{1 / 2}=0.1-2.5$ for 1 yr of operation, depending on the photon spectral index. A NEMO-generation 
detector, with a bin size $\Delta \Omega \sim 0.3 \times 0.3$ (Riccobene et al. 2002), would have an S/N roughly 3 times larger for the same integration time. The effect of neutrino oscillations may reduce the flux by a factor of 3 at Earth (Bilenky, Giunti, \& Grimus 1999). However, for some possible values of the photon spectral index, this signal could be detected in a reasonable time span. The possible starburst neutrino spectra producing the previously quoted $\mathrm{S} / \mathrm{N}$ are plotted, together with an upper limit for the atmospheric neutrino background with vertical incidence, in Figure $1 b .^{5}$

\section{CONCLUDING REMARKS}

Through hadronic interactions in their central regions, nearby starburst galaxies can generate high-energy $\gamma$-ray emission detectable at Earth. Proton illumination of the inner winds of massive stars in a high-density CR environment may result in $\mathrm{TeV}$ sources without strong counterparts at $\mathrm{MeV}-\mathrm{GeV}$ energies. The high number of early-type stars with strong stellar winds in this kind of galaxy might produce a strong collective effect, providing the setting for accelerating protons up to multi$\mathrm{TeV}$ energies. In addition, we have found that starbursts might also be potential sources for kilometer-scale neutrino telescopes within reasonable integration times, depending on the photon spectral index. The detection-or nondetection-of the neutrino signal may be an essential piece of evidence in the determination of the origin of the high-energy radiation.

Gamma-rays from neutral pion decays will be produced not

\footnotetext{
${ }^{5}$ Regrettably, the South Pole location of the IceCube telescope would render its observation of NGC 253 nearly impossible; only neutrinos going through Earth can be identified. The smaller northern telescope ANTARES $\left(0.1 \mathrm{~km}^{2}\right.$; Becherini et al. 2002) would need much longer integration times to be able to distinguish NGC 253 from the background, if at all possible. Nevertheless, the analysis presented here can be directly applied to M82, a northern sky starburst at the same distance as NGC 253.
}

only in the stellar winds at the core of the starburst but also in the diffuse matter among the stars. If we model the starburst region as a disk with a radius of $\sim 300 \mathrm{pc}$ and a thickness of $\sim 70 \mathrm{pc}$, where the average density of $\mathrm{H}$ atoms in the diffuse interstellar medium is $\sim 300 \mathrm{~cm}^{-3}$ (Paglione et al. 1996), we get that the expected flux above $1 \mathrm{TeV}$ is $F(E>1 \mathrm{TeV}) \simeq$ $3.9 \times 10^{-13}$ photons $\mathrm{cm}^{-2} \mathrm{~s}^{-1}$, for an average CR enhancement of $s \sim 1000$ (see Torres et al. 2002 for details of calculation). This means an additional diffuse contribution of $\sim 3 \times 10^{38}$ ergs $\mathrm{s}^{-1}$ to the total $\mathrm{TeV} \gamma$-ray luminosity of the starburst. In most of our models, this diffuse contribution is only a small fraction of the total $\pi^{0}$-decay $\gamma$-ray emission, which is dominated by interactions occurring in the stellar winds.

Finally, it is important to remember that the CANGAROO observations are consistent with an extended source, whose size might be even bigger than the galaxy itself. If this is true, it would imply that there might be another component generating TeV radiation, related with the Galactic halo. This component might probably be the result of inverse Compton scattering of cosmic-ray background photons by $\mathrm{TeV}$ electrons accelerated at the superwind. In such case, since the interactions occur in the Thomson regime, the spectrum should be harder than that produced by hadrons in the center of the starburst.

Comments by L. Anchordoqui, P. Benaglia, Y. M. Butt, C. Mauche, M. M. Kaufman-Bernadó, M. Pohl, and an anonymous referee are gratefully acknowledged. G. E. R.'s research is mainly supported by Fundación Antorchas, CONICET (under grant PIP 0430/98), and ANPCT (PICT 03-04881). The work of D. F. T. was performed under the auspices of the US Department of Energy (NNSA), by University of California's Lawrence Livermore National Laboratory under contract W-7405-Eng-48. His research was done while he was a visiting scientist at SISSA (Italy) and a visiting professor at the University of Barcelona (Spain), to which he gratefully acknowledges their hospitality.

\section{REFERENCES}

Aharonian, F., et al. 2002, A\&A, 393, L37

Alvarez-Muñiz, J., \& Halzen, F. 2002, ApJ, 576, L33

Anchordoqui, L. A., Goldberg, H., \& Torres, D. F. 2002, preprint (astro-ph/ 0209546)

Anchordoqui, L. A., Romero, G. E., \& Combi, J. A. 1999, Phys. Rev. D, 60, 103001

Anchordoqui, L. A., et al. 2003, ApJ, in press (hep-ph/0211231)

Becherini, Y., et al. 2002, preprint (hep-ph/0211173)

Beck, R., Carilli, C. L., Holdaway, M. L., \& Klein, U. 1994, A\&A, 292, 409

Benaglia, P., Romero, G. E., Stevens, I. R., \& Torres, D. F. 2001, A\&A, 366, 605

Berezinskii, V. S., Bulanov, S. V., Dogiel, V. A., Ginzburg, V. L., \& Ptuskin, V. S. 1990, Astrophysics of Cosmic Rays (Amsterdam: North-Holland), chap. 9

Bhattacharya, D., et al. 1994, ApJ, 437, 173

Bilenky, S. M., Giunti, C., \& Grimus, W. 1999, Prog. Part. Nucl. Phys., 43, 1

Blom, J. J., Paglione, T. A., \& Carramiñana, A. 1999, ApJ, 516, 744

Butt, Y. M., et al. 2003, ApJ, submitted

Bykov, A. M. 2001, Space Sci. Rev., 99, 317

Bykov, A. M., \& Fleishman, G. D. 1992a, MNRAS, 255, 269

1992b, Soviet Astron. Lett., 18, 95

Cassé, M., \& Paul, J. A. 1980, ApJ, 237, 236

Chen, W., White, R. L., \& Bertsch, D. 1996, A\&AS, 120, 423

Clark, J. S., \& Negueruela, I. 2002, A\&A, 396, L25

de Vaucouleurs, G. 1978, ApJ, 224, 710

Forbes, D. A., et al. 1993, ApJ, 406, L11

Gaisser, T. K. 1990, Cosmic Rays and Particle Physics (Cambridge: Cambridge Univ. Press)

Gaisser, T. K., Halzen, F., \& Stanev, T. 1995, Phys. Rep., 258, 173 (erratum 271, 355 [1996])

Georganopoulos, M., Kirk, J. G., \& Mastichiadis, A. 2001, ApJ, 561, 111
Heckman, T. M., Armus, L., \& Miley, G. K. 1990, ApJS, 74, 833

Itoh, C., et al. 2002, A\&A, 396, L1

Jokipii, J. R., Kóta, J., \& Merényi, E. 1993, ApJ, 405, 782

Karle, A., et al. 2002, preprint (astro-ph/0209556)

Keto, E., Hora, J. L., Fazio, G. G., Hoffman, W., \& Deutsch, L. 1999, ApJ, 518,183

Kóta, J., \& Jokipii, J. R. 1983, ApJ, 265, 573

Lamers, H. J. G. L. M., \& Cassinalli, J. P. 1999, Introduction to Stellar Winds (Cambridge: Cambridge Univ. Press)

Lang, K. R. 1999, Astrophysical Formulae, Vol. 1 (Berlin: Springer)

Lipari, P. 1993, Astropart. Phys., 1, 195

McCarthy, P. J., Heckman, T., \& van Breugel, W. 1987, AJ, 93, 264

Montmerle, T. 1979, ApJ, 231, 95

Paglione, T. A. D., Marscher, A. P., Jackson, J. M., \& Bertsch, D. L. 1996, ApJ, 460, 295

Ptak, A., Serlemitsos, P., Yaqoob, T., Mushotzky, R., \& Tsuru, T. 1997, AJ, 113,1286

Quenby, J. J., Lockwood, J. A., \& Webber, W. R. 1990, ApJ, 365, 365

Riccobene, G., et al. 2002, in Methodical Aspects of Underwater/Ice Neutrino Telescopes, ed. R. Wischnewski (Hamburg: DESY), 61

Rieke, G. H., et al. 1980, ApJ, 238, 24

Romero, G. E., Benaglia, P., \& Torres, D. F. 1999, A\&A, 348, 868

Stecker, F. W. 1979, ApJ, 228, 919

Suchkov, A., Allen, R. J., \& Heckman, T. M. 1993, ApJ, 413, 542

Torres, D. F., Romero, G. E., Dame, T. M., Combi, J. A., \& Butt, Y. M. 2002 , preprint (astro-ph/0209565)

Ulvestad, J. S., \& Antonucci, R. R. J. 1997, ApJ, 488, 621

Volkova, L. V. 1980, Soviet J. Nucl. Phys., 31, 784

Watson, A. M., et al. 1996, AJ, 112, 534

White, R. L. 1985, ApJ, 289, 698

White, R. L., \& Chen, W. 1992, ApJ, 387, L81 